\title{
Radius properties for analytic and p-valently starlike functions
}

Neslihan Uyanik ${ }^{1}$ and Shigeyoshi Owa ${ }^{2^{*}}$

\footnotetext{
* Correspondence: owa@math. kindai.ac.jp

${ }^{2}$ Department of Mathematics, Kinki University, Higashi-Osaka, Osaka 577-8502, Japan

Full list of author information is available at the end of the article
}

\section{Abstract}

Let $\mathcal{A}_{p}$ be the class of functions $\mathrm{f}(z)$ which are analytic in the open unit disk $\mathbb{U}$ and satisfy $\frac{z^{p}}{f(z)} \neq 0(z \in \mathcal{U})$. Also, let $\mathcal{S}_{p}^{*}(\alpha)$ denotes the subclass of $\mathcal{A}_{p}$ consisting of $f(z)$ which are $p$-valently starlike of order $\alpha(0 \leqq \alpha<p)$. A new subclass $\mathcal{U}_{p}(\lambda)$ of $\mathcal{A}_{p}$ is introduced by

$$
\left|z^{2}\left(\frac{z^{p-1}}{f(z)}-\frac{1}{z}\right)^{\prime}\right| \leqq \lambda \quad(z \in \mathbb{U})
$$

for some real $\lambda>0$. The object of the present paper is to consider some radius properties for $f(z) \in \mathcal{S}_{p}^{*}(\alpha)$ such that $\delta^{-p} f(\delta z) \in \mathcal{U}_{p}(\lambda)$.

2010 Mathematics Subject Classification: Primary 30C45.

Keywords: Analytic, radius problem, Cauchy-Schwarz inequality, $p$-valently starlike of order $a$.

\section{Introduction}

Let $\mathcal{A}_{p}$ be the class of functions $f(z)$ of the form

$$
f(z)=z^{p}+\sum_{n=p+1}^{\infty} a_{n} z^{n} \quad(p=1,2,3, \ldots)
$$

which are analytic in the open unit disk $\mathbb{U}=\{z \in \mathbb{C}:|z|<1\}$ and satisfy

$$
\frac{z^{p}}{f(z)}=1+\sum_{n=p+1}^{\infty} b_{n} z^{n-p} \neq 0 \quad(z \in \mathbb{U})
$$

For $f(z) \in \mathcal{A}_{p}$, we say that $f(z)$ belongs to the class $\mathcal{U}_{p}(\lambda)$ if it satisfies

$$
\left|z^{2}\left(\frac{z^{p-1}}{f(z)}-\frac{1}{z}\right)^{\prime}\right| \leqq \lambda \quad(z \in \mathbb{U})
$$

for some real number $\lambda>0$.

Let us consider a function $f_{\delta}(z)$ given by

$$
f_{\delta}(z)=\frac{z^{p}}{(1-z)^{\delta}} \quad(\delta \in \mathbb{R})
$$


Then, we can write that

$$
f_{\delta}(z)=\frac{z^{p}}{1+\sum_{n=1}^{\infty} a_{n} z^{n}}
$$

with

$$
a_{n}=(-1)^{n}\left(\begin{array}{l}
\delta \\
n
\end{array}\right)
$$

and

$$
\begin{aligned}
\left|z^{2}\left(\frac{z^{p-1}}{f_{\delta}(z)}-\frac{1}{z}\right)^{\prime}\right| & =\left|\sum_{n=1}^{\infty}(n-1) a_{n} z^{n}\right| \\
& <\sum_{n=1}^{\infty}(n-1)\left|a_{n}\right| .
\end{aligned}
$$

Thus, if $\delta=2$, then

$$
\left|z^{2}\left(\frac{z^{p-1}}{f_{2}(z)}-\frac{1}{z}\right)^{\prime}\right|<1 .
$$

This shows that $f_{2}(z) \in \mathcal{U}_{p}(\lambda)$ for $\lambda \geqq 1$.

If $\delta=3$, then we have that

$$
\left|z^{2}\left(\frac{z^{p-1}}{f_{3}(z)}-\frac{1}{z}\right)^{\prime}\right|<5
$$

Which shows that $f(z) \in \mathcal{U}_{p}(\lambda)$ for $\lambda \geqq 5$.

Further, if $\delta=4$, then

$$
\left|z^{2}\left(\frac{z^{p-1}}{f_{4}(z)}-\frac{1}{z}\right)^{\prime}\right|<11
$$

which shows that $f(z) \in \mathcal{U}_{p}(\lambda)$ for $\lambda \geqq 11$.

If $p=1$, then $f(z) \in \mathcal{U}_{1}(\lambda)$ is defined by

$$
\left|z^{2}\left(\frac{1}{f(z)}-\frac{1}{z}\right)^{\prime}\right| \leqq \lambda \quad(z \in \mathbb{U})
$$

for some real number $\lambda>0$. Note that (1.5) is equivalent to

$$
\left|f^{\prime}(z)\left(\frac{z}{f(z)}\right)^{2}-1\right| \leqq \lambda \quad(z \in \mathbb{U}) .
$$

Therefore, this class $\mathcal{U}_{1}(\lambda)$ was considered by Obradović and Ponnusamy [1]. Further-more, this class was extended as the class $\mathcal{U}\left(\beta_{1}, \beta_{2} ; \lambda\right)$ by Shimoda et al. [2]. 
Let $\mathcal{S}_{p}^{*}(\alpha)$ denotes the subclass of $\mathcal{A}_{p}$ consisting of $f(z)$ which satisfy

$$
\operatorname{Re}\left(\frac{z f^{\prime}(z)}{f(z)}\right)>\alpha \quad(z \in \mathbb{U})
$$

for some real $\alpha(0 \leqq \alpha<p)$.

A function $f(z) \in \mathcal{S}_{p}^{*}(\alpha)$ is said to be $p$-valently starlike of order $\alpha$ in $\mathbb{U}$ (cf. Robertson [3]).

\section{Coefficient inequalities}

For $f(z) \in \mathcal{A}_{p}$, we consider the sufficient condition for $f(z)$ to be in the class $\mathcal{U}_{p}(\lambda)$.

Lemma 1 If $f(z) \in \mathcal{A}_{p}$ satisfies

$$
\sum_{n=p+2}^{\infty}(n-p-1)\left|b_{n}\right| \leqq \lambda,
$$

then $f(z) \in \mathcal{U}_{1}(\lambda)$.

Proof We note that

$$
\begin{aligned}
\left|z^{2}\left(\frac{z^{p-1}}{f(z)}-\frac{1}{z}\right)^{\prime}\right| & =\left|\sum_{n=p+1}^{\infty}(n-p-1) b_{n} z^{n-p}\right| \\
& <\sum_{n=p+1}^{\infty}(n-p-1)\left|b_{n}\right| .
\end{aligned}
$$

Therefore, if

$$
\sum_{n=p+1}^{\infty}(n-p-1)\left|b_{n}\right|=\sum_{n=p+2}^{\infty}(n-p-1)\left|b_{n}\right| \leqq \lambda,
$$

then $f(z) \in \mathcal{U}_{p}(\lambda)$.

Example 1 If we consider a function $f(z) \in \mathcal{A}_{p}$ given by

$$
\frac{z^{p}}{f(z)}=1+b_{p+1} z+\sum_{n=p+2}^{\infty} \frac{\lambda e^{i \varphi}}{(n-p)(n-p-1)^{2}} z^{n-p} \neq 0 \quad(z \in \mathbb{U})
$$

with

$$
b_{n}=\frac{\lambda e^{i \varphi}}{(n-p)(n-p-1)^{2}}(\lambda>0, \varphi \in \mathbb{R})
$$

for $n \geqq p+2$, then we see that

$$
\begin{aligned}
\sum_{n=p+2}^{\infty}(n-p-1)\left|b_{n}\right| & =\sum_{n=p+2}^{\infty} \frac{\lambda e^{i \varphi}}{(n-p)(n-p-1)} \\
< & \lambda \sum_{n=p+2}^{\infty}\left(\frac{1}{n-p-1}-\frac{1}{n-p}\right)=\lambda .
\end{aligned}
$$


Thus, this function $f(z)$ satisfies the inequality (2.1). Also, we see that

$$
\begin{aligned}
\left|z^{2}\left(\frac{z^{p-1}}{f(x)}-\frac{1}{z}\right)^{\prime}\right| & =\left|\sum_{n=p+2}^{\infty} \frac{\lambda e^{i \varphi}}{n-p-1)(n-p)} z^{n-p}\right| \\
& <\lambda \sum_{n=p+2}^{\infty}\left(\frac{1}{n-p-1}-\frac{1}{n-p}\right)=\lambda .
\end{aligned}
$$

Therefore, we say that $f(z) \in \mathcal{U}_{p}(\lambda)$.

Next, we discuss the necessary condition for the class $\mathcal{S}_{p}^{*}(\alpha)$.

Lemma 2 If $f(z) \in \mathcal{S}_{p}^{*}(\alpha)$ satisfies

$$
\frac{z^{p}}{f(z)}=1+\sum_{n=p+1}^{\infty} b_{n} z^{n-p} \neq 0 \quad(z \in \mathbb{U})
$$

with $b_{n}=\left|b_{n}\right| \mathrm{e}^{i(n-p) \theta}(n=p+1, p+2, p+3, \ldots)$, then

$$
\sum_{n=p+1}^{\infty}(n+\alpha-2 p)\left|b_{n}\right| \leqq p-\alpha .
$$

Proof Let us define the function $F(z)$ by

$$
F(z)=\frac{z^{p}}{f(z)}=1+\sum_{n=p+1}^{\infty} b_{n} z^{n-p} .
$$

It follows that

$$
\begin{aligned}
& \operatorname{Re}\left(\frac{z f^{\prime}(z)}{f(z)}\right)=\operatorname{Re}\left(p-\frac{z F^{\prime}(z)}{F(z)}\right) \\
& =\operatorname{Re}\left(\frac{p-\sum_{n=p+1}^{\infty}(n-2 p) b_{n} z^{n-p}}{1+\sum_{n=p+1}^{\infty} b_{n} z^{n-p}}\right) \\
& =\operatorname{Re}\left(\frac{p-\sum_{n=p+1}^{\infty}(n-2 p)\left|b_{n}\right| e^{i(n-p) \theta} z^{n-p}}{1+\sum_{n=p+1}^{\infty}\left|b_{n}\right| \mathrm{e}^{i(n-p) \theta} z^{n-p}}\right)>\alpha
\end{aligned}
$$

for $z \in \mathbb{U}$. Letting $z=|z| \mathrm{e}^{-i \theta}$, we have that

$$
\frac{p-\sum_{n=p+1}^{\infty}(n-2 p)\left|b_{n}\right||z|^{n-p}}{1+\sum_{n=p+1}^{\infty}\left|b_{n}\right||z|^{n-p}}>\alpha \quad(z \in \mathbb{U}) .
$$

If we take $|z| \rightarrow 1^{-}$, we obtain that

$$
\frac{p-\sum_{n=p+1}^{\infty}(n-2 p)\left|b_{n}\right|}{1+\sum_{n=p+1}^{\infty}\left|b_{n}\right|} \geqq \alpha
$$

which implies that

$$
\sum_{n=p+1}^{\infty}(n+\alpha-2 p)\left|b_{n}\right| \leqq p-\alpha .
$$

Remark 1 If we take $p=1$ in Lemmas 1 and 2, then we have that 
(i) $f(z) \in \mathcal{A}_{1}, \sum_{n=2}^{\infty}(n-2)\left|b_{n}\right| \leqq \lambda \Rightarrow f(z) \in \mathcal{U}_{1}(\lambda)$

and

(ii) $f(z) \in \mathcal{S}^{*}(\alpha), \quad\left|b_{n}\right|=\left|b_{n}\right| e^{i(n-1) \theta} \Rightarrow \sum_{n=2}^{\infty}(n+\alpha-2)\left|b_{n}\right| \leqq 1-\alpha$.

\section{Radius problems}

Our main result for the radius problem is contained in

Theorem 1 Let $f(z) \in \mathcal{S}_{p}^{*}(\alpha)(p-1 \leqq \alpha<p)$ with

$$
\frac{z^{p}}{f(z)}=1+\sum_{n=p+1}^{\infty} b_{n} z^{n-p} \neq 0 \quad(z \in \mathbb{U}) .
$$

and $b_{n}=\left|b_{n}\right| \mathrm{e}^{i(n-p) \theta}(n=p+1, p+2, p+3, \ldots)$. If $\delta \in \mathbb{C}(|\delta|<1)$, then $\frac{1}{\delta^{p}} f(\delta z)$ belongs to the class $\mathcal{U}_{p}(\lambda)$ for $0<|\delta| \leqq\left|\delta_{0}(\lambda)\right|$, where $\left|\delta_{0}(\lambda)\right|$ is the smallest positive root of the equation

$$
|\delta|^{2} \sqrt{1-\alpha}-\left(1-|\delta|^{2}\right) \lambda=0,
$$

that is,

$$
\left|\delta_{0}(\lambda)\right|=\sqrt{\frac{\lambda}{\lambda+\sqrt{1-\alpha}}} .
$$

\section{Proof Since}

$$
f(\delta z)=\delta^{p} z^{p}+\sum_{n=p+1}^{\infty} a_{n} \delta^{n} z^{n},
$$

we have that

$$
\frac{z^{p}}{\frac{1}{\delta^{p}} f(\delta z)}=1+\sum_{n=p+1}^{\infty} b_{n} \delta^{n-p} z^{n-p} .
$$

In view of Lemma 1 , we have to show that

$$
\sum_{n=p+2}^{\infty}(n-p-1)\left|b_{n}\right||\delta|^{n-p} \leqq \lambda .
$$

Note that $f(z) \in \mathcal{S}_{p}^{*}(\alpha)$ satisfies

$$
\left|b_{n}\right| \leqq \frac{p-\alpha}{n+\alpha-2 p}<1 \quad(p-1 \leqq \alpha<p) .
$$


Applying Cauchy-Schwarz inequality, we obtain that

$$
\begin{aligned}
\left.\sum_{n=p+2}^{\infty}(n-p-1)\left|b_{n} \|\right| \delta\right|^{n-p} & \leqq\left(\sum_{n=p+2}^{\infty}(n-p-1)\left|b_{n}\right|^{2}\right)^{\frac{1}{2}}\left(\sum_{n=p+2}^{\infty}(n-p-1)|\delta|^{2(n-p)}\right)^{\frac{1}{2}} \\
& \leqq\left(\sum_{n=p+2}^{\infty}(n-p-1)|\delta|^{2(n-p)}\right)^{\frac{1}{2}} \sqrt{p-\alpha} .
\end{aligned}
$$

Let $|\delta|^{2}=x$. Then, we have that

$$
\begin{aligned}
\sum_{n=p+2}^{\infty}(n-p-1) x^{n-p} & =x^{2}\left(\sum_{n=p+2}^{\infty}(n-p-1) x^{n-p-2}\right) \\
& =x^{2}\left(\sum_{n=p+2}^{\infty} x^{n-p-1}\right)^{\prime} \\
& =x^{2}\left(\sum_{n=1}^{\infty} x^{n-1}\right) \\
& =\frac{x^{2}}{(1-x)^{2}} .
\end{aligned}
$$

This gives us that

$$
\sum_{n=p+2}^{\infty}(n-p-1)\left|b_{n}\right||\delta|^{n-p} \leqq \frac{|\delta|^{2} \sqrt{p-\alpha}}{1-|\delta|^{2}} .
$$

Let us define the function $h(|\delta|)$ by

$$
h(|\delta|)=|\delta|^{2} \sqrt{p-\alpha}-\left(1-|\delta|^{2}\right) \lambda .
$$

Then, $h(|\delta|)$ satisfies $h(0)=-\lambda<0$ and $h(1)=\sqrt{p-\alpha}>0$. Indeed, we have that $h$ $\left(\left|\delta_{0}(\lambda)\right|\right)=0$ for

$$
0<\left|\delta_{0}(\lambda)\right|=\sqrt{\frac{\lambda}{\lambda+\sqrt{p-\alpha}}}<1 .
$$

This completes the proof of the theorem.

Corollary 1 Let $f(z) \in \mathcal{S}_{1}^{*}(\alpha) \quad(0 \leqq \alpha<1)$ with

$$
\frac{z}{f(z)}=1+\sum_{n=2}^{\infty} b_{n} z^{n-1} \neq 0 \quad(z \in \mathbb{U})
$$

and $b_{n}=\left|b_{n}\right| \mathrm{e}^{i(n-1) \theta}(n=2,3,4, \ldots)$. If $\delta \in \mathbb{C}(|\delta|<1)$, then $\frac{1}{\delta} f(\delta z)$ belongs to the class $\mathcal{U}_{1}(\lambda)$ for $0<|\delta| \leqq\left|\delta_{0}(\lambda)\right|$, where $\left|\delta_{0}(\lambda)\right|$ is the smallest positive root of the equation

$$
|\delta|^{2} \sqrt{1-\alpha}-\left(1-|\delta|^{2}\right) \lambda=0,
$$


that is,

$$
\left|\delta_{0}(\lambda)\right|=\sqrt{\frac{\lambda}{\lambda+\sqrt{1-\alpha}}} .
$$

Remark 2 In view of (3.2), we define the function $g(\lambda)$ by

$$
g(\lambda)=\left|\delta_{0}(\lambda)\right|=\sqrt{\frac{\lambda}{\lambda+\sqrt{p-\alpha}}} .
$$

Then, we have that

$$
g^{\prime}(\lambda)=\frac{1}{2} \sqrt{\frac{p-\alpha}{\lambda(\lambda+\sqrt{p-\alpha})^{3}}}>0
$$

for $\lambda>0$. Therefore, $\left|\delta_{0}(\lambda)\right|$ given by (3.2) is increasing for $\lambda>0$.

Remark 3 If we put $\alpha=p-\frac{1}{2}$ in Theorem 1 , then

$$
\left|\delta_{0}(\lambda)\right|=\sqrt{\frac{2 \lambda}{2 \lambda+\sqrt{2}}} .
$$

Therefore, if we consider $\lambda=\frac{1}{2}$, then we see that

$$
\left|\delta_{0}\left(\frac{1}{2}\right)\right|=\sqrt{\frac{1}{1+\sqrt{2}}}=0.64359 \ldots
$$

and if we make $\lambda=5$, then we have that

$$
\left|\delta_{0}(5)\right|=\sqrt{\frac{10}{10+\sqrt{2}}}=0.93600 \ldots
$$

\section{Author details}

${ }^{1}$ Department of Mathematics, Kazim Karabekir Faculty of Education, Atatürk University, 25240 Erzurum, Turkey

²Department of Mathematics, Kinki University, Higashi-Osaka, Osaka 577-8502, Japan

\section{Authors' contributions}

QF carried out the main part of this article. All authors read and approved the final manuscript.

\section{Competing interests}

The authors declare that they have no competing interests.

Received: 28 June 2011 Accepted: 7 November 2011 Published: 7 November 2011

\section{References}

1. Obradović, M, Ponnusamy, S: Radius properties for subclasses of univalent functions. Analysis. 25, $183-188$ (2005). doi:10.1524/anly.2005.25.3.183

2. Shimoda, Y, Hayami, T, Owa, S: Notes on radius properties of certain univalent functions. Acta Univ Apul 377-383 (2009). (Special Issue)

3. Robertson, MS: On the theory of univalent functions. Ann Math. 37, 374-408 (1936). doi:10.2307/1968451

doi:10.1186/1029-242X-2011-107

Cite this article as: Uyanik and Owa: Radius properties for analytic and p-valently starlike functions. Journal of Inequalities and Applications 2011 2011:107. 